\title{
Epithelial markers in the diagnosis of nasopharyngeal carcinoma: an immunocytochemical study
}

\author{
BA GUSTERSON,${ }^{*}$ DIANA P MITCHELL,$\dagger$ MJ WARBURTON,${ }^{*}$ RL CARTER $\dagger$
}

From the * Ludwig Institute for Cancer Research (London Branch), Royal Marsden Hospital, Sutton, Surrey, the †Department of Histopathology, Royal Marsden Hospital, Sutton, Surrey, and the Institute of Cancer Research and Royal Marsden Hospital, Clifton Avenue, Belmont, Sutton, Surrey

SUMMARY Immunocytochemical stains for three epithelial cell markers-keratin, epithelial membrane antigen (EMA) and carcinoembryonic antigen (CEA)-have been examined on paraffin-embedded material from 14 patients with nasopharyngeal carcinoma (NPC). Tumour cells staining positively for keratin were found in all cases and for EMA in eight; two tumours contained CEA-positive cells. Seven cases of Hodgkin's disease and 24 non-Hodgkin's lymphomas were uniformly negative. Keratin is the most reliable epithelial marker for identifying NPC and excluding lymphoma. The regular finding of stainable keratin in non-keratinising and $\underset{\infty}{5}$ anaplastic NPC supports the view that NPC is a homogeneous group exhibiting variable degrees of squamous differentiation.

Antibodies to keratins have been shown to be of diagnostic value. in the assessment of the epithelial nature of both primary and metastatic poorly differentiated neoplasms. ${ }^{12}$ Anti-keratin antibodies have proved of particular value in distinguishing thymomas from lymphomas ${ }^{3}$ and as tools to study the cellular heterogeneity of both normal and malignant epithelial cells in both the lung ${ }^{4}$ and the breast. ${ }^{5}$ Another epithelial marker, epithelial membrane antigen (EMA), has also proved to be a useful reagent in the diagnostic dilemma of the undifferentiated tumour. ${ }^{6}$

In this paper we have studied the potential usefulness of these epithelial markers-keratin, epithelial membrane antigen and carcinoembryonic antigen-as diagnostic aids to differentiate between lymphomas and nasopharyngeal carcinomas.

\section{Material and methods}

\section{IMMUNOCYTOCHEMICAL REAGENTS}

Keratin was extracted from human carcinomas by the method of Sun and Green.' Antisera were raised by dissolving $1 \mathrm{mg}$ of keratin in $500 \mu \mathrm{l} 1 \mathrm{M}$ urea and $5 \mu \mathrm{l}$ of mercaptoethanol in phosphate-buffered saline, preparing an emulsion with $1 \mathrm{ml}$ Freund's complete adjuvant, and injecting it subcutaneously into the nuchal region of rabbits at monthly inter- vals. The resultant antisera gave a single immunoprecipitation band on Ouchterlony diffusion against purified keratin. Details of antibodies to epithelial membrane antigen (EMA) and carcinoembryonic $\frac{2}{2}$ antigen (CEA) have been previously described. .9 Second antibodies were obtained by affinity pur- $\frac{O}{3}$ ification of a sheep antirabbit gamma globulin, and conjugated to alkaline phosphatase using glutaraldehyde. ${ }^{10}$

\section{STAINING PROCEDURE}

A detailed account of the method used has been reported. ${ }^{11}$

Sections were cut from formalin-fixed paraffin wax-embedded material at approx $4 \mu \mathrm{m}$ and 음 dewaxed in xylene. The sections were treated with $D$ $15 \%$ acetic acid for $15 \mathrm{~min}$ to block endogenous alkaline phosphatase activity, incubated with the N appropriate antiserum diluted $1 / 100$ for $90 \mathrm{~min}$, washed and incubated with the enzyme-conjugated 0 second antibody for a further $90 \mathrm{~min}$. The stain was $\mathrm{\omega}$ developed using AS:B1 phosphoric acid plus Fast Red TR. The slides were counterstained with Mayer's haemalum and mounted in buffered glyce- $\mathbb{D}$ rin jelly.

Specificity of staining was confirmed by absorption of each antiserum with the appropriate antigen.

\section{Results}

The patterns of staining with keratin, EMA and 
Patterns of immunocytochemical staining for epithelial markers in nasopharyngeal carcinomas and in lymphomas

\begin{tabular}{llll}
\hline & \multicolumn{1}{l}{ Incidence of positive markers } \\
\cline { 2 - 4 } & Keratin & $\begin{array}{l}\text { Epithelial } \\
\text { membrane } \\
\text { antigen }\end{array}$ & $\begin{array}{l}\text { Carcinoembryonic } \\
\text { antigen }\end{array}$ \\
\hline $\begin{array}{l}\text { Nasopharyngeal carcinomas, primary } \mathrm{n}=8 \\
\text { Nasopharyngeal carcinomas, metastatic } \mathrm{n}=6\end{array}$ & 8 & 0 & 2 \\
$\begin{array}{l}\text { Non-Hodgkin's lymphoma } \\
\text { Hodgkin's disease } \mathrm{n}=7\end{array}=24$ & 6 & 4 & 0 \\
\hline
\end{tabular}

*Comprise histiocytic (8), poorly differentiated lymphocytic (6), immunoblastic (5), well differentiated lymphocytic (3), Burkitt (2). Tumours classified according to a modified Rappaport system. ${ }^{.9}$

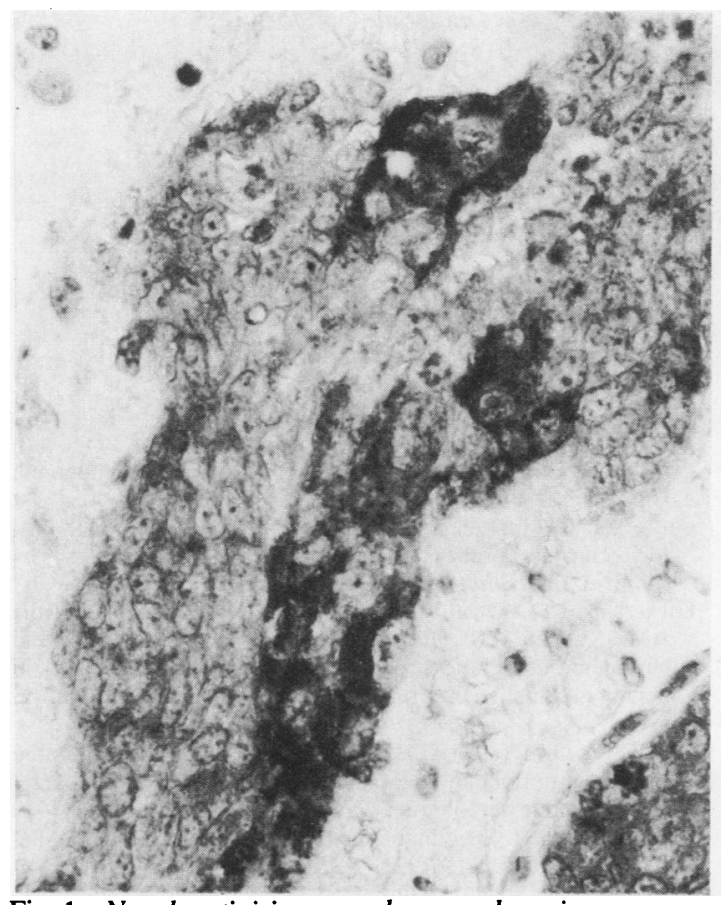

Fig. 1 Non-keratinising nasopharyngeal carcinoma stained with antikeratin antibodies. There is strong positive staining of all tumour cells with strong staining of focal areas. Stroma is negative. $\times 300$

CEA in 14 patients with nasopharyngeal carcinomas are summarised in the Table: control data from 31 patients with lymphomas are also included. Positive staining was confined to NPC, the lymphomas examined being uniformly negative. All NPC contained keratin-positive tumour cells, and eight tumours also contained EMA-positive elements. CEA-stained tumour cells were seen in only two cases. Keratin staining was, predictably, most marked in tumours with keratin pearls but it was also demonstrated in non-keratinised and undifferentiated forms. Positively-staining cells showed a characteristically patchy distribution in the tumours, and staining in individual cells was confined to the cytoplasm (Figs. 1 and 2). EMA-positive tumour cells showed a similar patchy distribution with staining concentrated along cell borders or more diffusely in the cytoplasm. In the undifferentiated NPC group the staining was weaker and focal but in all cases positive cells were identified.

\section{Discussion}

The recognition of primary and metastatic NPC can be difficult, ${ }^{12-14}$ despite the clarified diagnostic criteria proposed in the recent WHO classification of these tumours ${ }^{15}$ the continuing problem is the distinction between anaplastic NPC and lymphoma. Electron microscopy may be helpful ${ }^{14}$ but appropriately fixed tissues are often not taken at the time of biopsy. Immunocytochemical studies of epithelial cell markers can be readily performed on formalinfixed and paraffin wax-embedded tissues, and provide a useful diagnostic aid. Keratin staining in NPC, briefly noted by Costa ${ }^{14}$ has now been described in more detail in a recent survey of 40 cases by Madri and Barwick. ${ }^{16}$ Our results confirm the value of keratin staining in the recognition of NPC; we also show that staining with two other epithelial markers-EMA and CEA-is less reliable. When considering the general diagnostic problem of differentiating between anaplastic carcinoma and large cell lymphoma, epithelial membrane antigen is more reliable than the anti-keratin antiserum. ${ }^{20}$ In cases of poorly differentiated squamous cell carcinomas however, keratin is usually demonstrable. The fact that keratin is regularly demonstrated in the nonkeratinised and anaplastic forms of NPC strengthens the view that these tumours are a homogeneous group exhibiting varying degrees of squamoid differentiation. ${ }^{1718}$

\section{Addendum}

In a separate study, one of us (RLC) has investigated EMA - and CEA - staining in biopsies of 


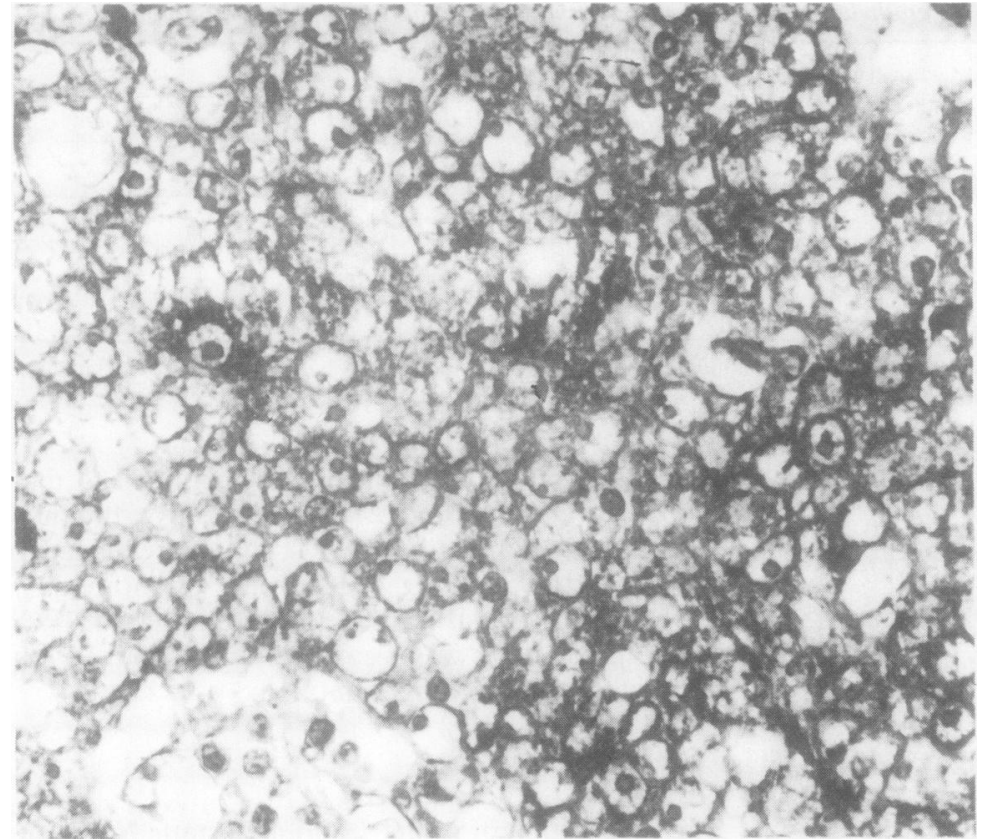

Fig. 2 Undifferentiated nasopharyngeal carcinoma positively stained with antikeratin antibodies. $\times 450$ nasopharyngeal carcinoma (NPC) from Guangxi, a high-incidence region in the People's Republic of China. The work was carried out in collaboration with Dr Zeng, Dr Li and Dr G de Thé. Among 15 examples of NPC, nine stained positively with EMA and only one with CEA. Local uninvolved mucosa was frequently positive for both antigens (EMA $8 / 10$, CEA 4/10). Mucosal staining was also positive in most of 15 control biopsies from patients with chronic nasopharyngeal inflammation (EMA 15/15, CEA 9/15). An association was noted in both tumour and control biopsies between positive staining for EMA and CEA and local chronic inflammation. Scattered foci of mild mucosal dysplasia, present in both series, did not "mark" with EMA or CEA in any distinctive fashion.

We are indebted to Dr A Levene for access to stored tissue blocks, Dr MG Ormerod for providing the antibodies to epithelial membrane antigen and carcinoembryonic antigen and to Mr G Cowley for providing the conjugate. RLC acknowledges support from the Medical Research Council.

\section{References}

' Schlegel R, Banks-Schlegel S, McLeod JA, Pinkus GS. Immunoperoxidase localisation of keratin in human neoplasms. Am J Pathol 1980;101:41-50.

: Sieinski W, Dorsett B, Ioachim HL. Identification of prekeratin by immunofluorescence staining in the differential diagnosis of tumours. Hum Pathol 1981;12:452-8.

${ }^{3}$ Battifora H, Sun T-T, Bahu RM, Rao S. The use of antikeratin antiserum as a diagnostic tool: thymoma versus lymphoma. Hum Pathol 1980;11:635-41.

${ }^{4}$ Gusterson B, Mitchell D, Warburton M, Sloane J. Immunohistochemical localisation of keratin in human lung tumours. Virchows Arch (Pathol Anat) 1982;394:269-77.

${ }^{5}$ Gusterson BA, Warburton MJ, Mitchell D, Ellison M, Neville AM, Rudland PS. Distribution of myoepithelial cells and basement membrane proteins in the normal breast and in benign and malignant breast disease. Cancer Res 1982;42: (in press).

- Sloane JP, Ormerod MG. Distribution of epithelial membrane antigen in normal and neoplastic tissues and its value in diagnostic tumour pathology. Cancer 1981;47:1786-95.

${ }^{7}$ Sun TT, Green H. Keratin filaments of cultured human epithelial cells. J Biol Chem 1978;253:2053-60.

${ }^{8}$ Heyderman E, Steele K, Ormerod MG. A new antigen of the epithelial membrane: its immunoperoxidase localisation in normal and neoplastic tissue. J Clin Pathol 1979;32:35-9.

9 Ormerod MG. Antigenic determinants of carcinoembryonic antigen. Scand J Immunol 1978;8 suppl 8;433-8.

${ }^{10}$ Avrameas S, Ternynck T. Peroxidase labelling antibody and Fab conjugates with enhanced intracellular penetration. N Immunochemistry 1971;8:1175-9.

"Mitchell DP, Gusterson BA. Simultaneous demonstration of N keratin and mucin. J Histochem Cytochem 1982;30:707-9.

${ }_{12}$ Micheau C. Anatomie pathologique et essori de classification des epitheliomas du nasopharynx. Bull Cancer 1975;62:277-86.

${ }^{13}$ Lennert K, Kaiserling E, Mazzonli T. Diagnosis and differential 0 diagnosis of lymphoepithelial carcinoma in lymph nodes: histological, cytological and electron-microscopic findings. In: de The G, Ito Y, Davis W, eds. Nasopharyngeal carcinoma: etiology and control. Lyon: International Agency for Research on Cancer, 1978:65-84.

${ }^{14}$ Costa J. The histopathological diagnosis of nasopharyngeal carcinoma. In: Grundmann E, Krueger GRF, Ablashi DV, eds. Cancer Campaign Volume 5: Nasopharyngeal carcinoma. Stuttgart, New York: Gustav Fischer, 1981:7-10.

is Shanmugaratnam K, Sobin LH. Histological typing of upper 
respiratory tract tumours. International classification of tumours No 14 Geneva: WHO, 1978.

${ }^{16}$ Madri JA, Barwick KW. An immunohistochemical study of nasopharyngeal neoplasms using keratin antibodies. $A m J$ Surg Pathol 1982;6:143-9.

${ }^{17}$ Shanmugaratnam K. Histological typing of nasopharyngeal carcinoma. In: de Thé G, Ito Y, Davis W, eds. Nasopharyngeal carcinoma: etiology and control. Lyon: International Agency for Research on Cancer, 1978:3-11.

${ }^{18}$ Weiland L. The histopathological spectrum of nasopharyngeal carcinoma. In: de Thé G, Ito Y, Davis W, eds. Nasopharyngeal carcinoma: etiology and control. Lyon: International Agency for Research on Cancer, 1978:41-50.
${ }^{19}$ Nathwani BN. A critical analysis of the classification of nonHodgkin's lymphomas. Cancer 1979;44:347-84.

${ }^{20}$ Sloane JP, Hughes F, Ormerod MG. An assessment of the value of epithelial membrane antigen and other epithelial markers in solving diagnostic problems in tumour pathology. Histochem $J$ 1982; (in press).

Requests for reprints to: Dr BA Gusterson, Ludwig Institute for Cancer Research (London Branch), Royal Marsden Hospital, Sutton, Surrey SM2 5PX, England. 